\title{
Schwarze Liste als Todesurteil
}

\section{Leana Cavelti}

Medizinstudentin im ersten Jahr, Universität Zürich

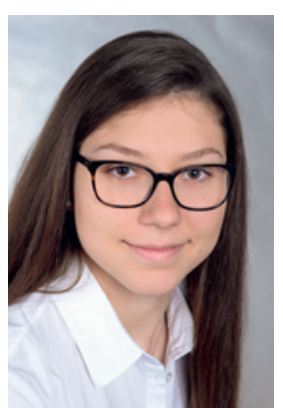

Es war an einem gemütlichen Abendessen, als ich mich mit meiner Familie über die Aufgabe meines EthikAufsatzes unterhielt. Ich war bezüglich meiner Themenwahl völlig ratlos, bis mein Vater mir von Herrn Meier erzählte (Name geändert). Sein Fall fesselte mich von Anfang an, und ich fing sofort an zu recherchieren. Schnell fand ich unzählige Artikel und Informationen dazu. Herr Meier war ein 50-jähriger HIV-Patient aus Chur. Weil er seine Krankenkassenprämien nicht mehr bezahlt hatte, stand er auf der schwarzen Liste des Kantons. Er bat seine Krankenkasse dennoch mehrmals, seine Therapien und Medikamente zu bezahlen. Beides blieb ihm jedoch verwehrt - auch dann noch, als Aids bereits ausgebrochen war. Inzwischen ist Herr Meier an einer Begleiterkrankung von Aids verstorben.

Dass die steigenden Prämien für viele Versicherte eine $\mathrm{zu}$ grosse finanzielle Belastung darstellen, ist nicht überraschend. Rund 150000 weitere Personen sind schweizweit auf einer solchen Liste vermerkt. Ärztinnen und Ärzte müssen diesen Patienten von Gesetzes wegen eine medizinische Versorgung verweigern und sie unbehandelt nach Hause schicken. Davon ausgenommen sind Notfallbehandlungen. Aber, wo fängt ein Notfall an und wo hört er auf? Sind unsere Ärztinnen und Ärzte in der Lage, eine solche Grenze in jedem Fall klar zu ziehen? Und ist die Behandlungsverweigerung, auch wenn sie rechtlich zulässig ist, ethisch zweifelsfrei vertretbar?

Anfänglich hatte ich Verständnis für das Verhalten seiner Krankenkasse. Der eigentliche Zweck der schwarzen Listen besteht darin, deutlich zu machen, dass Gesundheitsleistungen nicht zum Nulltarif erhältlich sind. Leider kommen aber nicht nur Bürger auf die Liste, die nicht zahlen wollen, sondern vermehrt auch diejenigen, die nicht zahlen können. Dies führt zu einer verstärkten Diskriminierung von sozial und wirtschaftlich schlechter gestellten Personen. Jeder Arzt steckt hier in einem Dilemma. Einerseits besteht die Pflicht, Patienten zu helfen und ihnen Leid zu ersparen, andererseits ist kein Spital daran interessiert, unnötige Pro-bono-Fälle zu übernehmen. Mir wurde deshalb schnell bewusst, dass Herrn Meiers Fall keine einfache Erklärung zulässt.

Als Erstes stellt sich mir die Frage: Was ist ein «Notfall»? Leider existiert hierfür keine einheitliche und präzise Umschreibung. In der Medizin wird letztlich jede Patientensituation als Notfall bezeichnet, die ohne medizinische Hilfe zu schweren langfristigen Schäden oder zum Tod führt. Im Falle von Herrn Meier wäre sofortige ärztliche Hilfe überlebenswichtig gewesen. Der Ausbruch von Aids hätte bei sofortiger Kostengutsprache seiner Krankenkasse weit hinausgezögert oder gar verhindert werden können. Trotzdem weigerte sich die Krankenkasse vehement, jegliche Auslagen zu übernehmen. Für mich ist es sehr schwierig, diesen Entscheid nachzuvollziehen. Denn wer garantiert mir, dass dieser Entschluss mit Bedacht und medizinisch begründet gefasst wurde? Und übernimmt eine ausreichend qualifizierte Person die Verantwortung für dieses Urteil?

Ein Grund für den Entscheid könnte im ethischen Grundprinzip der Gerechtigkeit liegen. Ist eine Übernahme von Herrn Meiers Kosten gegenüber bezahlenden Versicherten fair? Schliesslich könnte sich der Arzt an seiner Stelle nichtgelisteten Patienten widmen. Dadurch entsteht ein Konflikt zwischen dem Aspekt der Gerechtigkeit und dem Prinzip des Nichtschadens. Einerseits ist es gegenüber den Patienten, die ihre Prämienrechnung pünktlich bezahlen, nicht gerecht, sie zu vernachlässigen; andererseits darf Herr Meier durch die Verweigerung der Therapie nicht zu Schaden kommen. Ein Patient darf aber niemals aufgrund seiner wirtschaftlichen Lage priorisiert oder zurückgestellt werden. Ausschliesslich die Dringlichkeit seiner Erkrankung darf dabei eine Rolle spielen. Aufgrund des heutigen medizinischen Wissensstands hätte der Arzt in unserem Fall einer notfallmässigen Behandlung zustimmen müssen. Unglücklicherweise hatten die Entscheidungsträger für einen kurzen Augenblick über die schlechten Prognosen der Krankheit hinweggesehen, so dass Herr Meier mit seinem Leben bezahlen musste.

Ein weiteres Argument für den Entscheid könnte der damalige Gesundheitszustand des Patienten gewesen sein. Herrn Meiers Leben schien zu keinem Zeitpunkt ernsthaft bedroht, und so beurteilte es anscheinend auch die Krankenkasse, als sie ihre Zahlungen verweigerte. Für die erste Abweisung zum Zeitpunkt des positiven Befundes scheint mir dieses Argument noch halbwegs passabel. Halbwegs aus dem Grund, weil die Chancen auf ein beschwerdefreies Leben umso besser stehen, je früher die Therapie gestartet wird. Wenn 
wir uns aber in Erinnerung rufen, dass die zweite Abweisung nach Ausbruch von Aids erfolgte, wird das Argument mit Sicherheit hinfällig. Die Medikamente hätten seinem verbleibenden Leben durchaus noch Qualität schenken können.

Dieses eindrucksvolle Ereignis zeigt auf, welchen Weg unser Gesundheitssystem in Zukunft möglicherweise gehen wird: nämlich den einer Zweiklassenmedizin. Weil aber so das ethische Prinzip «Gleiches gleich und Ungleiches ungleich behandeln", wie es schon Aristoteles formuliert hat, verletzt wird, betrachte ich diese Stossrichtung höchst kritisch. Schwarze Listen verstärken diese Tendenz zusätzlich. Solange genügend finanzielle Mittel zur Verfügung stehen, scheint dies kein Problem zu sein. Mittellose Patienten hingegen geraten unter die Räder. Der gesetzlich verankerte diskriminierungsfreie Zugang zu ärztlichen Leistungen muss jedem Einwohner garantiert sein. Deswegen wage ich zu behaupten, dass Herr Meier Anrecht auf die kurativen Leistungen gehabt hätte. Unabhängig von der Tatsache, dass er auf der schwarzen Liste figurierte.

Die Auseinandersetzung mit Herrn Meiers Fall macht für mich deutlich, dass diese ethische Problemstellung schnellstmöglich eine nachhaltige Lösung erfordert.
Ein möglicher Ansatz liegt meines Erachtens in einer Änderung der Prämienzahlung. Statt wie bisher eine individuelle Rechnung begleichen zu müssen, würde jedem Arbeitnehmer eine lohnabhängige Grundprämie direkt vom Gehalt abgezogen werden. Erstens wäre so eine Grundversicherung für jeden Bürger gewährleistet. Zweitens wären kritische Diskussionen über Notfall- oder Nicht-Notfallpatienten überflüssig, und schliesslich würde die Praxis schwarzer Listen ein Ende finden. Allerdings ist meine Idee nicht neu. Das Parlament debattierte bereits über diese Angelegenheit, doch meines Wissens ist ein definitiver Entscheid noch ausstehend.

Ich bin zutiefst enttäuscht und erschrocken, dass sich ein solcher Fall in der reichen Schweiz abgespielt hat. Er führt mir vor Augen, wie stark unsere Medizin wirtschaftlich beeinflusst wird. Es geht nicht mehr nur darum, jemandem zu helfen, sondern vielmehr um den finanziellen Profit. Aber nicht nur wirtschaftliche Aspekte dürfen ärztliche Beschlüsse beeinflussen. Viel wichtiger ist der Einbezug von ethischen Betrachtungsweisen. Vor allem dann, wenn sich die Entscheidungen wie in unserem Fall in einer Grauzone bewegen, einem Bereich, wo kein Schwarz oder Weiss, Ja oder Nein existiert. 